\title{
The Error Estimates of the Interpolating Element-Free Galerkin Method for Two-Point Boundary Value Problems
}

\author{
J. F. Wang, ${ }^{1,2}$ S. Y. Hao, ${ }^{3}$ and Y. M. Cheng ${ }^{2}$ \\ ${ }^{1}$ Ningbo Institute of Technology, Zhejiang University, Ningbo 315100, China \\ ${ }^{2}$ Shanghai Institute of Applied Mathematics and Mechanics, Shanghai University, Shanghai 200072, China \\ ${ }^{3}$ School of Traffic and Transportation, Lanzhou Jiaotong University, Lanzhou 730070, China \\ Correspondence should be addressed to Y. M. Cheng; ymcheng@shu.edu.cn
}

Received 23 December 2013; Accepted 19 February 2014; Published 9 April 2014

Academic Editor: Miaojuan Peng

Copyright (c) 2014 J. F. Wang et al. This is an open access article distributed under the Creative Commons Attribution License, which permits unrestricted use, distribution, and reproduction in any medium, provided the original work is properly cited.

\begin{abstract}
The interpolating moving least-squares (IMLS) method is discussed in detail, and a simpler formula of the shape function of the IMLS method is obtained. Then, based on the IMLS method and the Galerkin weak form, an interpolating element-free Galerkin (IEFG) method for two-point boundary value problems is presented. The IEFG method has high computing speed and precision. Then error analysis of the IEFG method for two-point boundary value problems is presented. The convergence rates of the numerical solution and its derivatives of the IEFG method are presented. The theories show that, if the original solution is sufficiently smooth and the order of the basis functions is big enough, the solution of the IEFG method and its derivatives are convergent to the exact solutions in terms of the maximum radius of the domains of influence of nodes. For the purpose of demonstration, two selected numerical examples are given to confirm the theories.
\end{abstract}

\section{Introduction}

Conventional computational methods, such as the finite element method (FEM) and the boundary element method (BEM), cannot be applied well to some engineering problems. For the extremely large deformation and crack growth problems, the remeshing technique must be used. Meshless methods have been developed in recent years. The most important common feature of meshless methods is that the trial function is constructed from a set of nodes with no meshing at all. Then some complex problems, such as the large deformation and crack growth problems, can be simulated with the method without the remeshing techniques $[1,2]$.

Many kinds of meshless methods have been proposed, such as element-free Galerkin (EFG) method [3-5], complex variable meshless method [6-11], mesh-free reproducing kernel particle Ritz method [12], radial basis function (RBF) method [13], finite point method (FPM) [14-16], meshless local Petrov-Galerkin (MLPG) method [17], reproducing kernel particle method (RKPM) [18-22], meshless manifold method [23-29], boundary element-free method (BEFM) [30-40], and local boundary integral equation (LBIE) method [41, 42].

The element-free Galerkin (EFG) method is one of the most powerful meshless methods [3]. The EFG method can obtain a solution with high precision. Various problems have been successfully analyzed by the EFG method. By using the orthogonal function system with a weight function as the basis function, Zhang et al. presented the improved elementfree Galerkin method [43-48], which has high computational efficiency. By combining the complex variable moving leastsquares (CVMLS) approximation and the EFG method, Peng et al. presented the complex variable element-free Galerkin (CVEFG) method [49-56]. Compared with the conventional EFG methods, the CVEFG method has greater computational precision and efficiency.

The EFG method is constructed based on the moving least-squares (MLS) approximation. The shape function that is formed with MLS approximation can obtain a solution with high precision. However, a disadvantage of the MLS approximation is that its shape function does not satisfy the property 
of Kronecker $\delta$ function. Then the EFG method based on the MLS approximation cannot apply the essential boundary conditions directly and easily. The essential boundary conditions need to be introduced by additional approaches, such as Lagrange multipliers [3] and penalty methods [57]. However, for Lagrange multipliers, the corresponding discrete system will introduce additional unknowns which are not directly associated with the solution themselves. Furthermore, the banded structure of the matrix equation system is seriously worsened, as well as the conditioning properties. And, for penalty methods, the optimal value of penalty factor is hard to be set, which always affects the accuracy of the final solution.

To overcome this problem, Most and Bucher designed a regularized weight function with a regularization parameter $\varepsilon$, by which the MLS approximation can almost fulfill the interpolation with high accuracy [58]. Most and Bucher enhanced the regularized weighting function to obtain a true interpolation MLS approximation [59]. Another possible solution for this problem is the interpolating moving leastsquares (IMLS) method presented by Lancaster and Salkauskas [60]. The IMLS method is established based on the MLS approximation by using singular weight functions. The shape function of the IMLS method satisfies the property of Kronecker $\delta$ function. Thus the meshless methods based on the IMLS method can apply the essential boundary condition directly without any additional numerical effort. Based on the IMLS method, Kaljević and Saigal [61] presented an improved EFG method, in which the boundary condition is applied directly. Ren improved the expression of the shape function of the IMLS method and then presented the interpolating element-free Galerkin (IEFG) method and the interpolating boundary element-free (IBEFG) method for twodimensional potential and elasticity problems [62-65]. To overcome the singularity of the weight function in the IMLS method, Wang et al. presented the improved interpolating moving least-squares (IIMLS) method, in which nonsingular weight function is used [66-68]. In the IEFG method, the essential boundary conditions are applied directly and easily, and the number of unknown coefficients in the trial function of the IMLS method is less than that in the trial function of the MLS approximation. Therefore, the IEFG method based on the IMLS method has high computational efficiency and precision.

Error estimation for meshless method is certainly important to increase the reliability and reduce the cost of numerical computations in many engineering problems. Some error analyses have been done for the MLS approximation and the meshless method based on it [69-74]. Krysl and Belytschko studied the convergence of the continuous and discontinuous shape functions of the second-order elliptic partial differential equations [75]. Chung and Belytschko proposed the local and global error estimates using the difference between the values of the projected stress and these given directly by the EFG solution [76]. Dolbow and Belytschko studied the integration error [77]. Gavete et al. presented a procedure to estimate the error in elliptic equations and then proposed a posteriori error approximation $[78,79]$. R. J. Cheng and Y. M. Cheng studied the error estimate of the finite point method based on the MLS approximation [80] and the error estimates of element-free Galerkin method for potential and elasticity problems [81, 82]. For the IEFG method, since the essential boundary condition is applied directly, then the error estimate of the IEFG method is no doubt different from that of the EFG method. However, until now the error analysis of the IEFG method has not been seen in the recent literature.

Two-point boundary value problems occur in applied mathematics, theoretical physics, engineering, and optimization theory. Since it is usually impossible to obtain analytical solutions to two-point boundary value problems met in practice, these problems must be attacked by numerical methods. Many numerical methods have been proposed for the solutions of these problems, such as the Galerkin and collocation methods, boundary value method, variational iteration method, and meshless method based radial basis functions.

In this paper, the IMLS method is discussed in detail. The computation of the shape function of this paper is simpler than the corresponding expression presented by Lancaster and Salkauskas. Then based on the IMLS method of this paper and the Galerkin weak form, an IEFG method for two-point boundary value problems is presented. Since the shape function of the IMLS method satisfies the property of Kronecker $\delta$ function, then the IEFG method can apply the essential boundary condition directly. And as the number of the coefficients in the trial function of the IMLS method is less than that in the MLS approximation, then fewer nodes are selected in the entire domain in the IEFG method than in the conventional EFG method. Hence, the IEFG method has high computing speed and precision.

Then the error analysis of the IEFG method for two-point boundary value problems is presented. The convergence rates of the numerical solution and its derivatives of the IEFG method are presented. The theoretical results show that if the exact solution is smooth enough and the order of the polynomial basis functions is big enough, then the solution of the IEFG method and its derivatives are convergent to the exact solutions in terms of the maximum radius of the domains of influence of nodes. For the purpose of demonstration, some selected numerical examples are given to confirm the theory.

\section{Interpolating Moving Least-Squares Method}

Let $\mathbf{X}=\left\{\mathbf{x}_{1}, \mathbf{x}_{2}, \ldots, \mathbf{x}_{M}\right\}$ be a set of all nodes in the bounded domain $\Omega \subset R^{n}$, where $M$ is the number of nodes. The parameter $\rho_{I}$ denotes the radius of the domain of influence of node $\mathbf{x}_{I}$, and $\|\cdot\|$ denotes the Euclidean norm. The domain of influence of $\mathbf{x}_{I}$ is defined by

$$
\Omega_{I}=\left\{\mathbf{x} \mid\left\|\mathbf{x}-\mathbf{x}_{I}\right\| \leq \rho_{I}, \mathbf{x} \in \Omega\right\} .
$$

Let $u(\mathbf{x})$ be the function of the field variable defined in $\Omega$. The approximation function of $u(\mathbf{x})$ is denoted by $u^{h}(\mathbf{x})$. In this paper, the following weight function is used:

$$
w\left(\mathbf{x}, \mathbf{x}_{I}\right)=m_{I}(\mathbf{x})\left\|\frac{\mathbf{x}-\mathbf{x}_{I}}{\rho_{I}}\right\|^{-\alpha},
$$


where $m_{I}(\mathbf{x})=m\left(\mathbf{x}-\mathbf{x}_{I}\right) \in C^{l}(\Omega)$ satisfying $m_{I}(\mathbf{x})>0$ for $\left\|\mathbf{x}-\mathbf{x}_{I}\right\|<\rho_{I}$ and $m_{I}(\mathbf{x})=0$ for $\left\|\mathbf{x}-\mathbf{x}_{I}\right\| \geq \rho_{I}$. In general, $m_{I}(\mathbf{x})$ can be chosen to be any weight function of the MLS approximation.

For a given point $\mathbf{x}$, the inner product is defined as

$$
(f, g)_{\mathbf{x}}=\sum_{I=1}^{n} w\left(\mathbf{x}, \mathbf{x}_{I}\right) f\left(\mathbf{x}_{I}\right) g\left(\mathbf{x}_{I}\right), \quad \forall f, g \in C^{0}(\Omega),
$$

where $n$ is the number of nodes whose compact support domains cover $\mathbf{x}$.

Let $p_{0}(\mathbf{x}) \equiv 1$ and let $p_{1}(\mathbf{x}), \ldots, p_{m}(\mathbf{x})$ be given basis functions. Then a new set of basis functions will be generated from these given basis. Let

$$
\begin{aligned}
\tilde{p}_{0}(\mathbf{x} ; \overline{\mathbf{x}}) & =\frac{1}{\left[\sum_{I=1}^{n} w\left(\mathbf{x}, \mathbf{x}_{I}\right)\right]^{1 / 2}}, \\
\tilde{p}_{i}(\mathbf{x} ; \overline{\mathbf{x}}) & =p_{i}(\overline{\mathbf{x}})-\mathbf{S} p_{i}(\mathbf{x}), \quad i=1,2, \ldots, m,
\end{aligned}
$$

where $\mathbf{S}$ is a linear operator defined as

$$
\begin{gathered}
\mathbf{S} p_{i}(\mathbf{x})=\sum_{I=1}^{n} v\left(\mathbf{x}, \mathbf{x}_{I}\right) p_{i}\left(\mathbf{x}_{I}\right), \\
v\left(\mathbf{x}, \mathbf{x}_{I}\right)=\frac{w\left(\mathbf{x}, \mathbf{x}_{I}\right)}{\sum_{J=1}^{n} w\left(\mathbf{x}, \mathbf{x}_{J}\right)} .
\end{gathered}
$$

Define a local approximation as

$$
u^{h}(\mathbf{x}, \overline{\mathbf{x}})=\widetilde{p}_{0}(\mathbf{x} ; \overline{\mathbf{x}}) a_{0}(\mathbf{x})+\sum_{i=1}^{m} \widetilde{p}_{i}(\mathbf{x} ; \overline{\mathbf{x}}) a_{i}(\mathbf{x}),
$$

where $\overline{\mathbf{x}}$ is the point in the domain of influence of $\mathbf{x}$, and $a_{i}(\mathbf{x})(i=0,1, \ldots, m)$ are the unknown coefficients of basis functions.

Then define a functional as

$$
\kappa=\sum_{I=1}^{n} w\left(\mathbf{x}, \mathbf{x}_{I}\right)\left[u^{h}\left(\mathbf{x}, \mathbf{x}_{I}\right)-u_{I}\right]^{2},
$$

where $w\left(\mathbf{x}, \mathbf{x}_{I}\right)$ shown in (2) is a weight function with compact support, $\mathbf{x}_{I}$ are the nodes with domains of influence that cover the point $\mathbf{x}$, and $u_{I}=u\left(\mathbf{x}_{I}\right)$.

By minimizing the functional $\kappa$, we have

$$
\begin{gathered}
\left(u(\cdot)-u^{h}(\mathbf{x}, \cdot), \widetilde{p}_{0}\right)_{\mathbf{x}}=0, \\
\left(u(\cdot)-u^{h}(\mathbf{x}, \cdot), \widetilde{p}_{i}\right)_{\mathbf{x}}=0, \quad i=1,2, \ldots, m .
\end{gathered}
$$

From (4) and (10), we have

$$
\widetilde{p}_{0}(\mathbf{x} ; \overline{\mathbf{x}}) a_{0}(\mathbf{x})=\sum_{I=1}^{n} v\left(\mathbf{x}, \mathbf{x}_{I}\right) u=\mathbf{S} u .
$$

Then (11) reduces to

$$
\sum_{i=1}^{m} a_{i}(\mathbf{x})\left(\tilde{p}_{i}, \widetilde{p}_{j}\right)_{\mathbf{x}}=\left(u-\mathbf{S} u, \widetilde{p}_{j}\right)_{\mathbf{x}}, \quad j=1,2, \ldots, m .
$$

In [60], the unknown parameters $a_{i}(\mathbf{x})(i=1,2, \ldots, m)$ are solved from (13). In fact, (13) can be simplified.
If the weight function of (2) is used, $\forall \mathbf{x} \in \Omega$, it can be proved that there exists

$$
\left(\mathrm{S} u, \widetilde{p}_{i}\right)_{\mathbf{x}}=0, \quad i=1,2, \ldots, m .
$$

Then (13) can be simplified as

$$
\sum_{i=1}^{m} a_{i}(\mathbf{x})\left(\widetilde{p}_{i}, \widetilde{p}_{j}\right)_{\mathbf{x}}=\left(u, \widetilde{p}_{j}\right)_{\mathbf{x}}, \quad j=1,2, \ldots, m .
$$

Equation (15) is simpler than the expression (13) presented in [60] and can be rewritten as

$$
\mathbf{A}(\mathbf{x}) \mathbf{a}(\mathbf{x})=\mathbf{F}_{\mathbf{W}}(\mathbf{x}) \mathbf{u},
$$

where

$$
\begin{gathered}
\mathbf{a}^{T}(\mathbf{x})=\left(a_{1}(\mathbf{x}), a_{2}(\mathbf{x}), \ldots, a_{m}(\mathbf{x})\right), \\
\mathbf{u}^{T}=\left(u_{1}, u_{2}, \ldots, u_{n}\right), \\
\mathbf{A}(\mathbf{x})=\mathbf{F}_{\mathbf{W}}(\mathbf{x}) \mathbf{F}^{T}(\mathbf{x}), \\
\mathbf{F}(\mathbf{x})=\left[\begin{array}{cccc}
\tilde{p}_{1}\left(\mathbf{x} ; \mathbf{x}_{1}\right) & \tilde{p}_{1}\left(\mathbf{x} ; \mathbf{x}_{2}\right) & \cdots & \widetilde{p}_{1}\left(\mathbf{x} ; \mathbf{x}_{n}\right) \\
\widetilde{p}_{2}\left(\mathbf{x} ; \mathbf{x}_{1}\right) & \widetilde{p}_{2}\left(\mathbf{x} ; \mathbf{x}_{2}\right) & \cdots & \widetilde{p}_{2}\left(\mathbf{x} ; \mathbf{x}_{n}\right) \\
\vdots & \vdots & \ddots & \vdots \\
\tilde{p}_{m}\left(\mathbf{x} ; \mathbf{x}_{1}\right) & \tilde{p}_{m}\left(\mathbf{x} ; \mathbf{x}_{2}\right) & \cdots & \tilde{p}_{m}\left(\mathbf{x} ; \mathbf{x}_{n}\right)
\end{array}\right],
\end{gathered}
$$

and $\mathbf{F}_{\mathbf{W}}(\mathbf{x})=\left(\omega_{k J}(\mathbf{x})\right)_{m \times n}$ is a $m \times n$ matrix, and

$$
\omega_{k J}(\mathbf{x})= \begin{cases}w\left(\mathbf{x}, \mathbf{x}_{J}\right) \widetilde{p}_{k}\left(\mathbf{x} ; \mathbf{x}_{J}\right), & \mathbf{x} \neq \mathbf{x}_{J} \\ \sum_{I=1, I \neq J}^{n} w\left(\mathbf{x}_{J}, \mathbf{x}_{I}\right)\left[p_{k}\left(\mathbf{x}_{J}\right)-p_{k}\left(\mathbf{x}_{I}\right)\right], & \mathbf{x}=\mathbf{x}_{J} .\end{cases}
$$

From (16), we have

$$
\mathbf{a}(\mathbf{x})=\mathbf{A}^{-1}(\mathbf{x}) \mathbf{F}_{\mathbf{W}}(\mathbf{x}) \mathbf{u} .
$$

Then the local approximation function is obtained as

$$
u^{h}(\mathbf{x}, \overline{\mathbf{x}})=\mathbf{S} u+\sum_{i=1}^{m} a_{i}(\mathbf{x}) \widetilde{p}_{i}(\mathbf{x} ; \overline{\mathbf{x}}) .
$$

Thus the global interpolating approximation function of $u(\mathbf{x})$ can be obtained as

$$
u^{h}(\mathbf{x})=\mathbf{S} u+\sum_{i=1}^{m} a_{i}(\mathbf{x}) g_{i}(\mathbf{x}) \equiv \boldsymbol{\Phi}^{T}(\mathbf{x}) \mathbf{u},
$$

where $\boldsymbol{\Phi}^{T}(\mathbf{x})$ is the shape function vector as

$$
\begin{gathered}
\boldsymbol{\Phi}^{T}(\mathbf{x})=\mathbf{v}^{T}+\mathbf{p}^{T}(\mathbf{x}) \mathbf{A}^{-1}(\mathbf{x}) \mathbf{F}_{\mathbf{W}}(\mathbf{x}), \\
\mathbf{v}^{T}=\left(v\left(\mathbf{x}, \mathbf{x}_{1}\right), v\left(\mathbf{x}, \mathbf{x}_{2}\right), \ldots, v\left(\mathbf{x}, \mathbf{x}_{n}\right)\right), \\
\mathbf{p}^{T}(\mathbf{x})=\left(g_{1}(\mathbf{x}), g_{2}(\mathbf{x}), \ldots, g_{m}(\mathbf{x})\right), \\
g_{i}(\mathbf{x})=p_{i}(\mathbf{x})-\mathbf{S} p_{i}(\mathbf{x}) .
\end{gathered}
$$

Equation (25) is the shape function of the IMLS method, and then the IMLS method is presented. 
Equations (13) and (15) show that the computation of the shape function of this paper is simpler than the corresponding expression in [60]. The reduction of computational amount is related to the order of the operations. And, in any case, the calculation amount of the shape functions of this paper is at least $n^{2}$ multiplication operations less than that of the IMLS method in [60].

\section{Interpolating Element-Free Galerkin Method for Two-Point Boundary Value Problems}

Consider the following two-point boundary value problem:

$$
\begin{gathered}
-\frac{\mathrm{d}}{\mathrm{d} x}\left(p \frac{\mathrm{d} u}{\mathrm{~d} x}\right)+q \frac{\mathrm{d} u}{\mathrm{~d} x}+g u=f, \quad x \in \Gamma=(a, b), \\
u(a)=u(b)=0,
\end{gathered}
$$

where $p, q, g$, and $f$ are known sufficiently smooth functions and $p(x) \geq p_{\text {min }}>0 . \forall f \in C(\Gamma)$, suppose that problem (29) has a unique solution.

The Galerkin weak form of (29) is

$$
\int_{\Gamma} \delta u_{, x} p u, x \mathrm{~d} x+\int_{\Gamma} \delta u q u, x \mathrm{~d} x+\int_{\Gamma} \delta u g u \mathrm{~d} x=\int_{\Gamma} \delta u f \mathrm{~d} x,
$$

where $u_{, x}=\mathrm{d} u / \mathrm{d} x$.

From the IMLS method, the unknown solution $u(x)$ at arbitrary field point $x$ in the interval $\Gamma$ can be expressed as

$$
u(x) \approx \Phi^{T}(x) \mathbf{u}=\sum_{I=1}^{n} \Phi_{I}(x) u_{I},
$$

where $n$ is the number of nodes whose compact support domains cover the point $x$.

Substituting (31) into (30) yields

$$
\begin{array}{r}
\int_{\Gamma} \delta \mathbf{u}^{T} \boldsymbol{\Phi}_{, x} \boldsymbol{\Phi}_{, x}^{T} \mathbf{u} p \mathrm{~d} x+\int_{\Gamma} \delta \mathbf{u}^{T} \boldsymbol{\Phi} \boldsymbol{\Phi}_{, x}^{T} \mathbf{u} q \mathrm{~d} x \\
+\int_{\Gamma} \delta \mathbf{u}^{T} \boldsymbol{\Phi} \boldsymbol{\Phi}^{T} \mathbf{u} g \mathrm{~d} x=\int_{\Gamma} \delta \mathbf{u}^{T} \boldsymbol{\Phi} f \mathrm{~d} x .
\end{array}
$$

Because the nodal test function $\delta \mathbf{u}$ is arbitrary, the final discretized equation of (29) is obtained as

$$
\mathbf{K u}=\mathbf{F},
$$

where

$$
\begin{gathered}
\mathbf{K}=\int_{\Gamma} p \boldsymbol{\Phi}_{, x} \boldsymbol{\Phi}_{, x}^{T} \mathrm{~d} x+\int_{\Gamma} q \boldsymbol{\Phi} \boldsymbol{\Phi}_{, x}^{T} \mathrm{~d} x+\int_{\Gamma} g \boldsymbol{\Phi} \boldsymbol{\Phi}^{T} \mathrm{~d} x \\
\mathbf{F}=\int_{\Gamma} \boldsymbol{\Phi} f \mathrm{~d} x .
\end{gathered}
$$

Since the shape function of the IMLS method satisfies the property of Kronecker $\delta$ function, then the essential boundary conditions can be applied directly. Substituting the boundary conditions into (33) directly, we can obtain the unknowns at nodes by solving the linear equations (33).

To evaluate the integrals in (34), it is necessary to generate integration cells over the whole domain of the problem. These cells can be defined arbitrarily, but a sufficient number of quadrature points must be used to obtain a well-conditioned and nonsingular system of (33). In one dimension, one example of a cell structure is to set the quadrature cells equal to the intervals between the nodes. Once the cells and corresponding quadrature points are defined, the discrete equations are assembled by looping over each quadrature point.

The numerical procedure of IEFG method for two-point boundary value problems is listed as follows.

(1) Looping over background cells to determine all Gauss points to find out its location and weight.

(2) Looping over Gauss points for integration of (34) to

(a) determine the support domain for specified Gauss point and select neighboring nodes based on a defined criterion;

(b) compute shape function and its derivatives for each Gauss point;

(c) assemble the contribution of each Gauss point to form system equation.

(3) Enforcing essential (displacement) boundary conditions.

(4) Solving the system equation to obtain nodal displacements.

Thus the IEFG method is presented for two-point boundary value problems.

\section{Error Estimates}

In this section, the error analysis of the IEFG method for two-point boundary value problems is presented. The convergence rates of the numerical solution and its derivatives of the IEFG method are presented.

Let $\mathbf{z}=\left\{x_{1}, x_{2}, \ldots, x_{M}\right\}$ be a set of all nodes in the interval $[a, b]$, where $M$ is the number of nodes. Let $\varepsilon=$ $\min _{x_{I}, x_{J} \in \mathbf{z}, I \neq J}\left\{\left\|x_{I}-x_{J}\right\|\right\}$ and $\rho=\max _{x_{I} \in \mathbf{z}}\left\{\rho_{I}\right\}$, where $\rho_{I}$ is the radius of the domain of influence of node $x_{I}$. For a given $x$, $\rho_{x}$ denotes the maximum radius of the influence domains of nodes whose compact support domains cover $x$. And suppose that there exist constants $c_{\varepsilon}$ and $c_{I}$ such that $\rho \leq c_{\varepsilon} \varepsilon$ and $\rho \leq c_{I} \rho_{I}$, respectively.

Define the $L^{p}$ Lebesgue space as

$$
L^{p}(\Gamma):=\left\{f(x):\|f\|_{L^{p}(\Gamma)}<\infty\right\}, \quad 1 \leq p<\infty,
$$

where

$$
\|f\|_{L^{p}(\Gamma)}:=\left(\int_{\Gamma} f^{p}(x) \mathrm{d} x\right)^{1 / p} .
$$

The Sobolev space $H^{k}(\Gamma)$ is defined as

$$
H^{k}(\Gamma):=\left\{f \in L_{\mathrm{loc}}^{1}(\Gamma):\|f\|_{H^{k}(\Gamma)}<\infty\right\}
$$


where

$$
\|f\|_{H^{k}(\Gamma)}:=\left(\sum_{s \leq k}\left\|\frac{\mathrm{d}^{s} f(x)}{\mathrm{d} x^{s}}\right\|_{L^{2}(\Gamma)}\right)^{1 / 2} .
$$

Define

$$
\begin{array}{r}
H_{0}^{1}(\Gamma)=\left\{v \mid v \in H^{1}(\Gamma), v(a)=v(b)=0\right\}, \\
\Gamma=[a, b] .
\end{array}
$$

Then the variational problem in accordance with (29) is to find $u \in H_{0}^{1}(\Gamma)$ such that

$$
a(u, v)=(f, v), \quad \forall v \in H_{0}^{1}(\Gamma),
$$

where

$$
\begin{gathered}
a(u, v)=\int_{\Gamma} p u_{, x} v_{, x} \mathrm{~d} x+\int_{\Gamma} q u, x v \mathrm{~d} x+\int_{\Gamma} g u v \mathrm{~d} x, \\
(f, v)=\int_{\Gamma} f v \mathrm{~d} x .
\end{gathered}
$$

Suppose that the bilinear form $a(\cdot, \cdot)$ on the Sobolev space $H_{0}^{1}(\Gamma)$ is bounded and coercive; that is, there exist constants $\bar{\alpha}>0$ and $\bar{M}<\infty$ such that

$$
\begin{gathered}
|a(u, v)| \leq \bar{M}\|u\|_{H^{1}(\Gamma)}\|v\|_{H^{1}(\Gamma)}, \quad \forall u, v \in H_{0}^{1}(\Gamma), \\
a(v, v) \geq \bar{\alpha}\|v\|_{H^{1}(\Gamma)}^{2}, \quad \forall v \in H_{0}^{1}(\Gamma) .
\end{gathered}
$$

Since the shape function of the IMLS method satisfies the property of Kronecker $\delta$ function, then the finitedimensional solution space of the IEFG method for two-point boundary value problems is defined as

$$
V_{\rho}(\Gamma)=\left\{v \mid v \in \operatorname{span}\left\{\Phi_{I}(x), 1 \leq I \leq M\right\}, v(a)=v(b)=0\right\} .
$$

Then the IEFG method according to (29) is to find $u_{h} \in$ $V_{\rho}(\Gamma)$ such that

$$
a\left(u_{h}, v\right)=(f, v), \quad \forall v \in V_{\rho}(\Gamma) .
$$

Obviously, there exists $V_{\rho}(\Gamma) \subset H_{0}^{1}(\Gamma)$. Hence, the IEFG method for two-point boundary value problems has a unique solution. And the following theorem can be obtained.

Theorem 1. Suppose that $u$ is the solution of the variational problem (40) and $u_{h}$ is the solution of the IEFG method (44). Then there exist

(a) $a\left(u-u_{h}, v\right)=0, \forall v \in V_{\rho}(\Gamma)$;

(b) $a\left(u-u_{h}, u-u_{h}\right)=\inf _{v \in V_{\rho}(\Gamma)} a(u-v, u-v)$;

(c) $\left\|u-u_{h}\right\|_{H^{1}(\Gamma)} \leq C \inf _{v \in V_{\rho}(\Gamma)}\|u-v\|_{H^{1}(\Gamma)}$.

In fact, the approximation function of the IMLS method provides a linear operator $\mathscr{A}$ defined as

$$
\mathscr{A} u \equiv \mathbf{S} u+\sum_{i=1}^{m} a_{i}(x) g_{i}(x)=\boldsymbol{\Phi}^{T}(x) \mathbf{u}
$$

If $u \in H^{m+1}(\Gamma)$, then we have proved that there exist bounded function $C_{k}^{\prime}(x)$ and constant $C_{k}$ such that

$$
\begin{gathered}
\frac{\mathrm{d}^{k}}{\mathrm{~d} x^{k}} \Phi_{I}(x)=C_{k}^{\prime}(x) \rho_{x}^{-k}, \\
\|\mathscr{A} u-u\|_{H^{k}(\Gamma)} \leq C_{k} \rho^{m+1-k}\|u\|_{H^{m+1}(\Gamma)}, \\
0 \leq k \leq m .
\end{gathered}
$$

Let $\left\|u-u_{h}\right\|_{L}^{2}=a\left(u-u_{h}, u-u_{h}\right)$. Then the following error estimates of the energy norm and the $H^{1}$ norm can be obtained.

Theorem 2. Suppose that $u \in H^{m+1}(\Gamma)$. Let $u$ and $u_{h}$ be, respectively, the solutions of the problems (40) and (44). Then there exist $C_{1}$ and $C_{2}$, which are independent with the parameter $\rho$, such that

$$
\begin{gathered}
\left\|u-u_{h}\right\|_{L} \leq C_{1} \rho^{m}\|u\|_{H^{m+1}(\Gamma)}, \\
\left\|u-u_{h}\right\|_{H^{1}(\Gamma)} \leq C_{2} \rho^{m}\|u\|_{H^{m+1}(\Gamma)}
\end{gathered}
$$

Proof. From Theorem 1 and (47) we have

$$
\begin{aligned}
\left\|u-u_{h}\right\|_{L}^{2} & =a\left(u-u_{h}, u-u_{h}\right) \\
& =\inf _{v \in V_{\rho}(\Gamma)} a(u-v, u-v) \\
& \leq a(u-\mathscr{A} u, u-\mathscr{A} u) \\
& \leq M\|u-\mathscr{A} u\|_{H^{1}(\Gamma)}^{2} \leq C_{1} \rho^{2 m}\|u\|_{H^{m+1}(\Gamma)}^{2}, \\
\left\|u-u_{h}\right\|_{H^{1}(\Gamma)} & \leq C \inf _{v \in V_{\rho}(\Gamma)}\|u-v\|_{H^{1}(\Gamma)} \\
& \leq C\|\mathscr{A} u-u\|_{H^{1}(\Gamma)} \leq C_{2} \rho^{m}\|u\|_{H^{m+1}(\Gamma)} .
\end{aligned}
$$

Then this theorem holds.

By using the Aubin-Nitsche method, the following error estimates in the $L^{2}$ norm can be obtained.

Theorem 3. Suppose that $u \in H^{m+1}(\Gamma)$. Let $u$ and $u_{h}$ be, respectively, the solutions of the problems (40) and (44). Then there exists a constant $C$, which is independent with the parameter $\rho$, such that

$$
\left\|u-u_{h}\right\|_{L^{2}(\Gamma)} \leq C \rho^{m+1}\|u\|_{H^{m+1}(\Gamma)} .
$$

Proof. For $\forall g \in L_{2}(\Gamma)$, let $\varphi \in H_{0}^{1} \cap H^{2}$ be the solution to the adjoint problems

$$
a(\varphi, v)=(g, v), \quad \forall v \in H_{0}^{1}(\Gamma) .
$$

If the coefficients of the bilinear form $a(\cdot, \cdot)$ are sufficiently smooth, then there exists the following estimate:

$$
\|\varphi\|_{H^{2}(\Gamma)} \leq\|g\|_{L^{2}(\Gamma)} .
$$

Let $v=u-u_{h}$. Then we have

$$
a\left(\varphi, u-u_{h}\right)=\left(g, u-u_{h}\right) .
$$


For arbitrary $v_{h} \in V_{\rho}$, from Theorem 1, we have

$$
a\left(v_{h}, u-u_{h}\right)=0 .
$$

It follows from (53) and (54) that

$$
a\left(\varphi-v_{h}, u-u_{h}\right)=\left(g, u-u_{h}\right) .
$$
exists

In (55), if we let $\mathscr{V}_{\hbar}=\mathscr{A} \varphi$ and $g=u-u_{h}$, then there

$$
\begin{aligned}
\left\|u-u_{h}\right\|_{L^{2}(\Gamma)}^{2} & =\left(u-u_{h}, u-u_{h}\right) \\
& =a\left(\varphi-\mathscr{A} \varphi, u-u_{h}\right) \\
& \leq M\|\varphi-\mathscr{A} \varphi\|_{H^{1}(\Gamma)}\left\|u-u_{h}\right\|_{H^{1}(\Gamma)} \\
& \leq M C_{1} \rho\|\varphi\|_{H^{2}(\Gamma)} C_{2} \rho^{m}\|u\|_{H^{m+1}(\Gamma)} .
\end{aligned}
$$

From (52) and (56), we have

$$
\left\|u-u_{h}\right\|_{L^{2}(\Gamma)} \leq C \rho^{m+1}\|u\|_{H^{m+1}(\Gamma)} .
$$

Then this theorem is proved.

To study the error estimates of the high derivatives of the numerical solution of the IEFG method, we need to firstly prove the following inverse estimates of the function in the shape function space.

Theorem 4. Suppose that $\Phi_{I}(x)$ is defined by (25). Then $\forall v_{h}(x) \in \operatorname{span}\left\{\Phi_{I}(x), 1 \leq I \leq M\right\}$; there exists a constant $C$, which is independent with the parameter $\rho$, such that

$$
\left\|v_{h}(x)\right\|_{H^{k}} \leq C \rho^{n-k}\left\|v_{h}(x)\right\|_{H^{n}}, \quad 0 \leq k \leq m,-m \leq n \leq m .
$$

Proof. From (46), there exists a bounded function $C_{k}(x)$ independent with $\rho$ such that

$$
\partial^{k} v_{h}(x)=C_{k}(x) \rho_{x}^{-k}
$$

where $\partial^{k} v_{h}(x)=\left(\mathrm{d}^{k} / \mathrm{d} x^{k}\right) v_{h}(x)$.

It is obvious that there exist bounded functions $C_{1}(x)$ and $C_{2}(x)$ independent with $\rho$ such that

$$
C_{1}(x) \rho \leq \rho_{\mathbf{x}} \leq C_{2}(x) \rho .
$$

Then we have

$$
\begin{array}{r}
\left|v_{h}(x)\right|_{H^{k}}^{2}=\int_{\Gamma}\left[\partial^{k} v_{h}(x)\right]^{2} \mathrm{~d} x \leq \int_{\Gamma}\left[C_{k}(x) C_{2}(x) \rho^{-k}\right]^{2} \mathrm{~d} x \\
0 \leq k \leq m, \\
\left|v_{h}(x)\right|_{H^{n}}^{2}=\int_{\Gamma}\left[\partial^{n} v_{h}(x)\right]^{2} \mathrm{~d} x \geq \int_{\Gamma}\left[C_{k}(x) C_{2}(x) \rho^{-n}\right]^{2} \mathrm{~d} x . \\
0 \leq n \leq m .
\end{array}
$$

Then it follows from (61) that

$$
\left\|v_{h}(x)\right\|_{H^{k}} \leq C \rho^{n-k}\left\|v_{h}(x)\right\|_{H^{n}}, \quad 0 \leq k, n \leq m,
$$

where $C$ is independent with $\rho$.
From (62), we have

$$
\left\|v_{h}(x)\right\|_{H^{k}} \leq C \rho^{-k}\left\|v_{h}(x)\right\|_{H^{0}}, \quad 0 \leq k \leq m .
$$

And there exists

$$
\begin{aligned}
\left\|v_{h}(x)\right\|_{H^{0}}^{2} & \leq\left\|v_{h}(x)\right\|_{H^{n}}\left\|v_{h}(x)\right\|_{H^{-n}} \\
& \leq\left\|v_{h}(x)\right\|_{H^{n}} \cdot C \rho^{n}\left\|v_{h}(x)\right\|_{H^{0}}, \quad-m \leq n \leq 0 .
\end{aligned}
$$

From (63) and (64), we have

$$
\left\|v_{h}(x)\right\|_{H^{k}} \leq C \rho^{n-k}\left\|v_{h}(x)\right\|_{H^{n}}, \quad 0 \leq k \leq m,-m \leq n \leq 0 .
$$

Then from (62) and (65), this theorem holds.

By using the inverse estimates, the following error estimates of the high derivatives of the numerical solution can be obtained.

Theorem 5. Suppose that $u \in H^{m+1}(\Gamma)$. Let $u$ and $u_{h}$ be, respectively, the solutions of the problems (40) and (44). Then there exists a constant $C$ independent with $\rho$, such that

$$
\left\|u-u_{h}\right\|_{H^{s}(\Gamma)} \leq C \rho^{m+1-s}\|u\|_{H^{m+1}(\Gamma)}, \quad 0 \leq s \leq m .
$$

Proof. From Theorem 4 we have

$$
\left\|u_{h}-\mathscr{A} u\right\|_{H^{s}(\Gamma)} \leq C h^{1-s}\left\|u_{h}-\mathscr{A} u\right\|_{H^{1}(\Gamma)}, \quad 0 \leq s \leq m .
$$

Then it follows from Theorem 2 and (47) that

$$
\begin{aligned}
\left\|u_{h}-\mathscr{A} u\right\|_{H^{s}(\Gamma)} & \leq C h^{1-s}\left[\left\|u_{h}-u\right\|_{H^{1}(\Gamma)}+\|u-\mathscr{A} u\|_{H^{1}(\Gamma)}\right] \\
& \leq C h^{m+1-s}\|u\|_{H^{m+1}(\Gamma)} .
\end{aligned}
$$

There certainly exits

$$
\left\|u-u_{h}\right\|_{H^{s}(\Gamma)} \leq\|u-\mathscr{A} u\|_{H^{s}(\Gamma)}+\left\|\mathscr{A} u-u_{h}\right\|_{H^{s}(\Gamma)} .
$$

From (68), (69), and (47), we have

$$
\left\|u-u_{h}\right\|_{H^{s}(\Gamma)} \leq C \rho^{m+1-s}\|u\|_{H^{m+1}(\Gamma)} .
$$

Then this theorem holds.

\section{Numerical Examples}

In this section, two numerical examples are presented to show the applicability and the theoretical error estimates of the IEFG method of this paper. In our numerical computation, the nodes are arranged regularly, and the radius of the domain of influence of node $x_{I}$ is determined by $\rho_{I}=d_{\max } \cdot\left|x_{I}-x_{I-1}\right|$, where $d_{\max }$ is a positive scalar. The value of $d_{\max }$ must be chosen so that the solution of (22) exists. In our following examples, $d_{\max }=2.5$ and $\alpha=4$. Define the error norms as

$$
\begin{aligned}
\left|e_{k}\right| & \equiv\left\|\frac{\mathrm{d}^{k}}{\mathrm{~d} x^{k}} u_{h}(\mathbf{x})-\frac{\mathrm{d}^{k}}{\mathrm{~d} x^{k}} u(\mathbf{x})\right\|_{L^{2}(\Gamma)} \\
& =\left[\int_{\Gamma}\left(\frac{\mathrm{d}^{k}}{\mathrm{~d} x^{k}} u_{h}-\frac{\mathrm{d}^{k}}{\mathrm{~d} x^{k}} u\right)^{2} \mathrm{~d} \Gamma\right]^{1 / 2},
\end{aligned}
$$




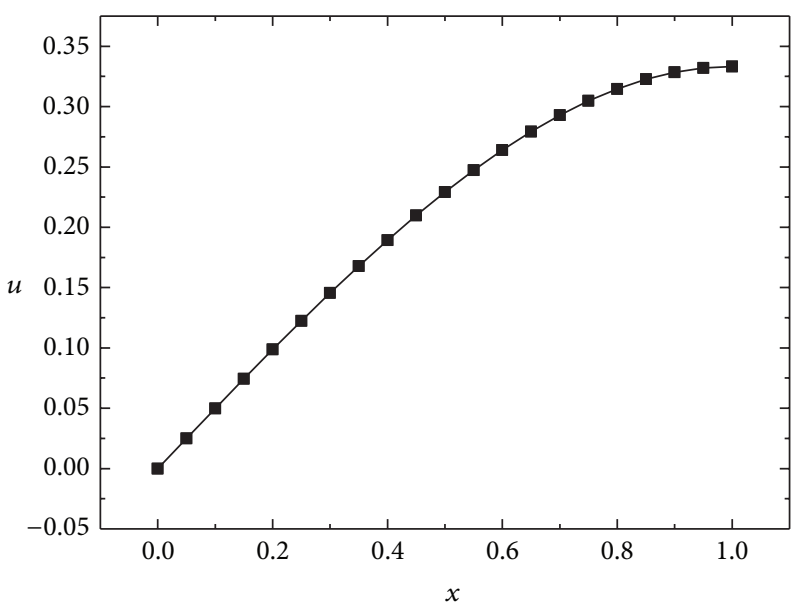

- Analytical

- Numerical

FIGURE 1: The analytical and numerical displacement.

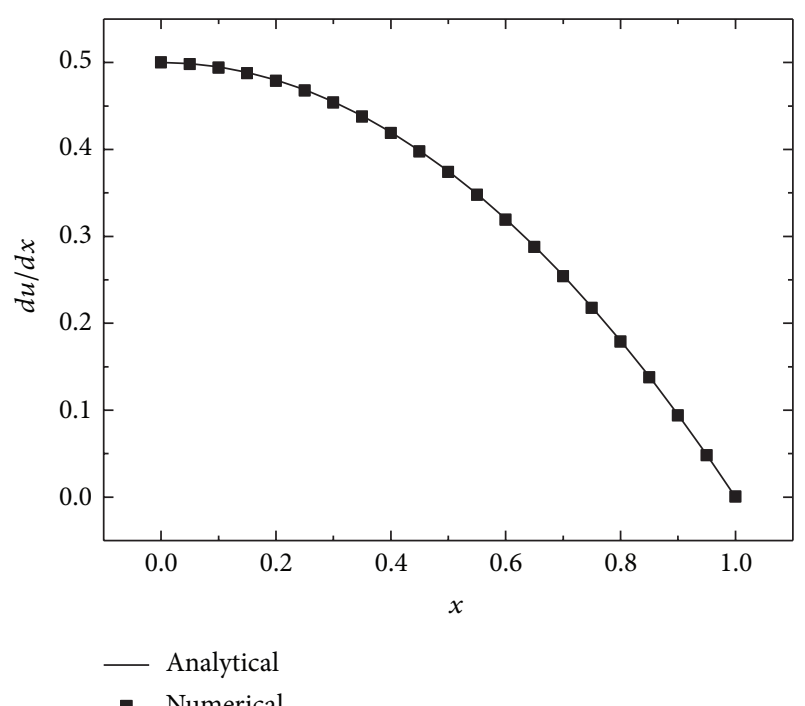

FIGURE 2: The analytical and numerical strain.

where $u$ and $u_{h}$ are, respectively, the analytical and numerical solutions. The integration in (71) is obtained numerically by fourth-order Gaussian quadrature. The $m_{I}(\mathbf{x})$ in (2) is chosen to be the cubic spline weight function.

The first example considered is a linear elastostatics problem. A one-dimensional bar of unit length is subjected to a body force of magnitude $x$. The displacement of the bar is fixed at the left end, and the right end is traction free. The bar has a constant cross sectional area of the unit value, and the elastic modulus is $E$. Then the equilibrium equation and boundary conditions of this problem are

$$
\begin{gathered}
E u_{, x x}+x=0, \quad 0<x<1, \\
u(0)=0, \quad u_{, x}(1)=0 .
\end{gathered}
$$

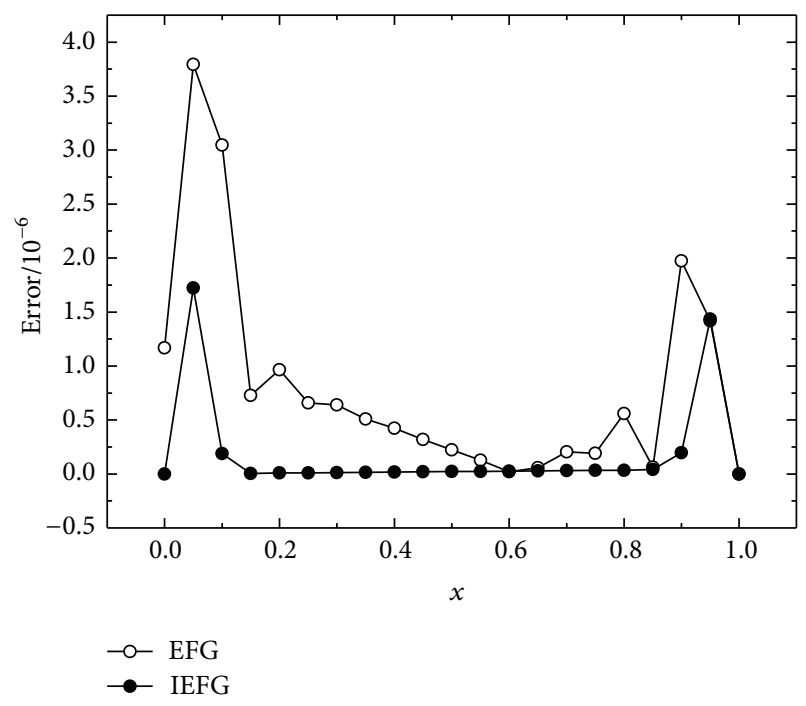

FIgURE 3: The absolute error of the EFG and IEFG methods.

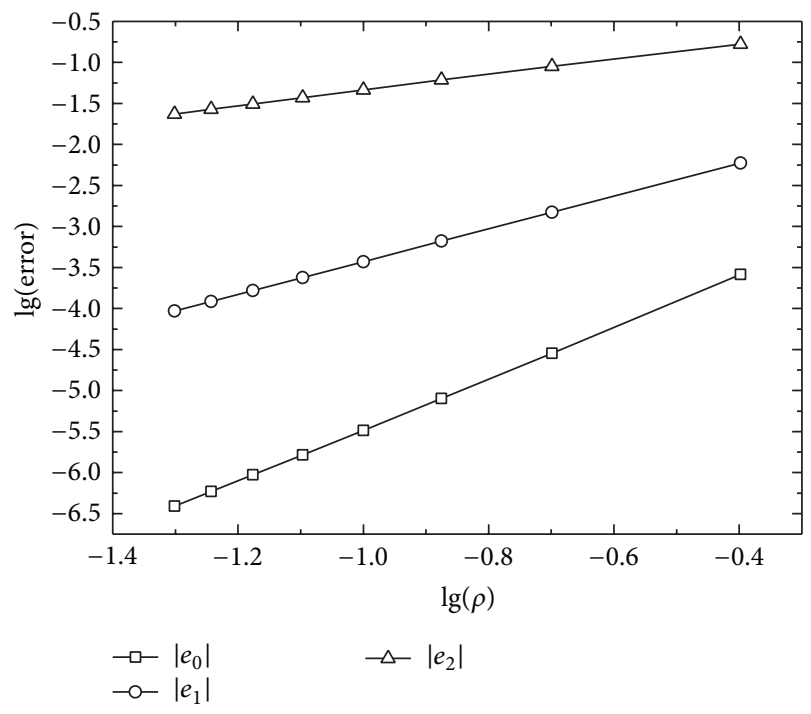

FIGURE 4: Error norms of $\left|e_{0}\right|,\left|e_{1}\right|$, and $\left|e_{2}\right|$ with quadratic basis functions.

The analytical solution to the above problem is

$$
u(x)=\frac{1}{E}\left(\frac{x}{2}-\frac{x^{3}}{6}\right) .
$$

Let $E=1$. Under the quadratic basis functions and 21 regular distributed nodes, the analytical and numerical solutions of the displacement and strains along the bar are shown, respectively, in Figures 1 and 2, where the numerical values of the IEFG method are in good agreement with the exact ones.

The absolute errors of the displacements obtained by the IEFG and EFG methods are shown in Figure 3. Here, the essential boundary conditions are enforced by the penalty method in the EFG method, and the penalty factor is chosen 


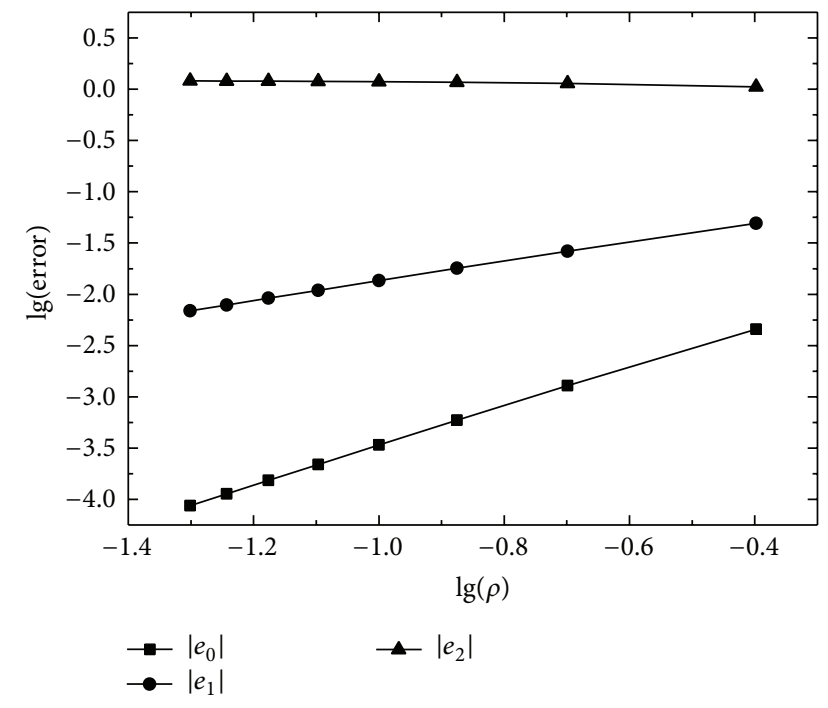

Figure 5: Error norms of $\left|e_{0}\right|,\left|e_{1}\right|$, and $\left|e_{2}\right|$ with linear basis functions.

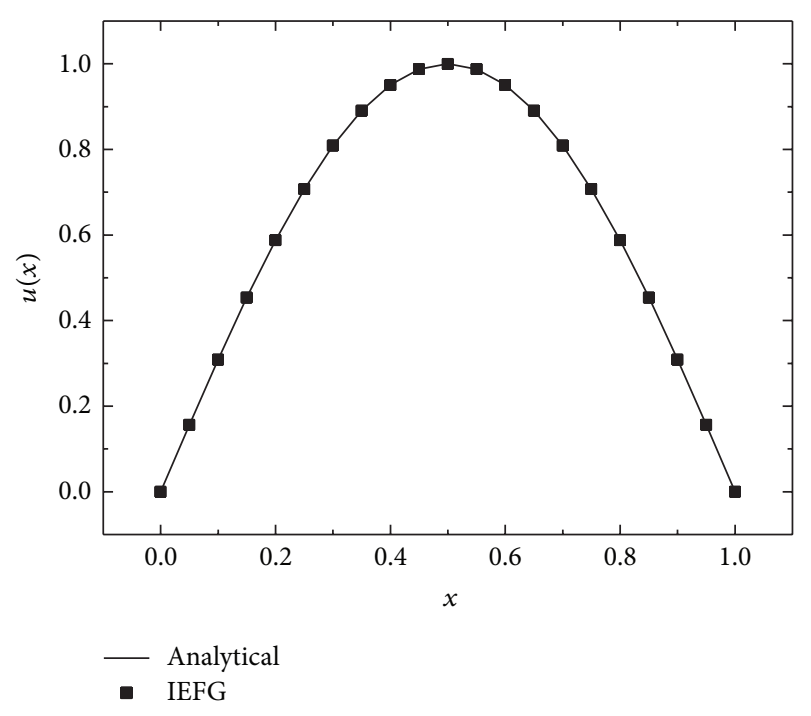

FIGURE 6: Analytical and numerical solutions of $u$.

to be $10^{8}$. The CPU times to obtain these results by using the IEFG and EFG methods are, respectively, $0.0267 \mathrm{~s}$ and $0.0253 \mathrm{~s}$. It can be seen that the IEFG method in this paper has higher precision than the EFG method under the similar CPU time.

The error norms of $\left|e_{0}\right|,\left|e_{1}\right|$, and $\left|e_{2}\right|$ under the quadratic and linear basis functions are, respectively, shown in Figures 4 and 5. The convergence rates of $\left|e_{0}\right|,\left|e_{1}\right|$, and $\left|e_{2}\right|$ with quadratic basis are, respectively, about 3,2 , and 1 , and the convergence rates with linear basis are 2,1 , and -0.1 . It is also shown that the second derivatives of the numerical solution of the IEFG method are not convergent to the exact values on the radius $\rho$ when the linear basis is used. It can be seen that these numerical results are in excellent agreement with the ones of the theories of the paper.

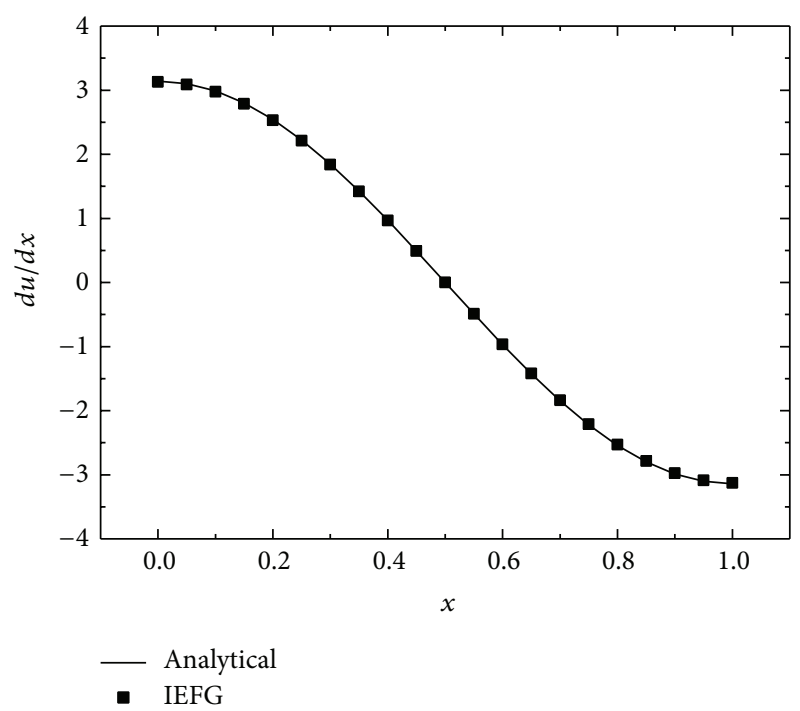

FIGURE 7: Analytical and numerical solutions of $u_{, x}$.

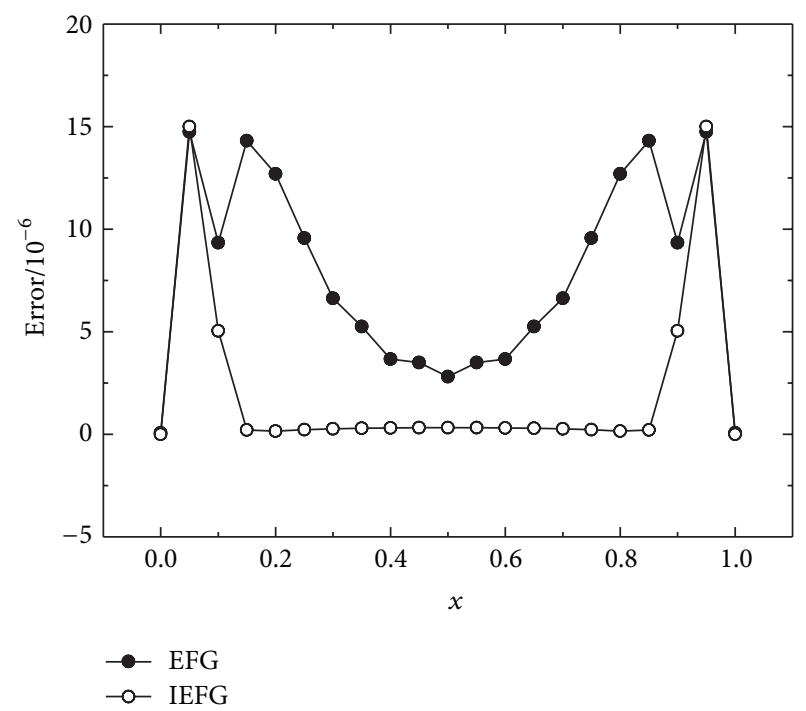

FIGURE 8: The absolute error of the EFG and IEFG methods.

The second example considers the following equilibrium equation:

$$
u_{, x x}+\pi^{2} u=2 \pi^{2} \sin (\pi x), \quad 0<x<1,
$$

with the boundary conditions

$$
u(0)=u(1)=0 .
$$

The analytical solution of this example is

$$
u(x)=\sin (\pi x) .
$$

When the quadratic basis functions and 21 regular distributed nodes are used, the analytical and numerical solutions of $u$ and $u_{, x}$ are shown, respectively, in Figures 6 and 7 , where the numerical values of the IEFG method are also in accordance well with the exact ones. 


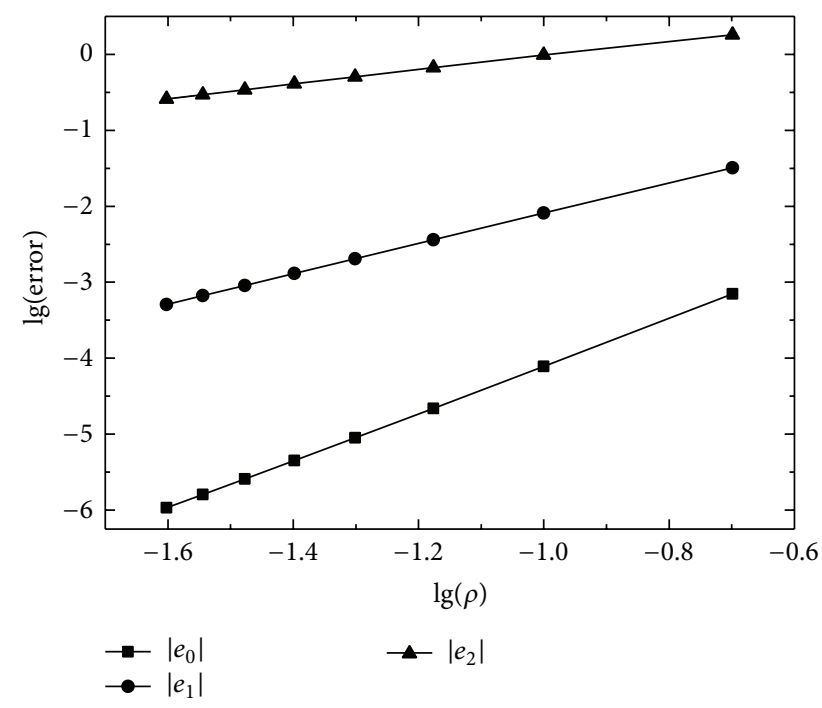

Figure 9: Error norms of $\left|e_{0}\right|,\left|e_{1}\right|$, and $\left|e_{2}\right|$ with quadratic basis functions.

The absolute errors of $u$ obtained by the IEFG and EFG methods are shown in Figure 8. In the EFG method, the cubic spline weight function is used, and the essential boundary conditions are enforced by the penalty method. The penalty factor is chosen to be $10^{10}$. The CPU times to obtain these results by the IEFG and EFG methods are, respectively, $0.0361 \mathrm{~s}$ and $0.0325 \mathrm{~s}$. Again, the IEFG method has higher precision than the EFG method under the similar CPU time.

The convergence rates of $\left|e_{0}\right|,\left|e_{1}\right|$, and $\left|e_{2}\right|$ are shown in Figures 9 and 10, respectively, with the quadratic and linear basis functions. The convergence rates of $\left|e_{0}\right|,\left|e_{1}\right|$, and $\left|e_{2}\right|$ with the quadratic basis are, respectively, 3,2 , and 1 . And the corresponding rates with linear basis are, respectively, 2,1 , and -0.004 . Figure 10 also shows that the second derivatives of the numerical solution of the IEFG method are not convergent to the exact values on the radius $\rho$ under the linear basis. It is also evident that these numerical results agree well with the ones of the theories of the paper.

\section{Conclusions}

In this paper, the IMLS method is discussed in detail. The computation of the shape function of this paper is simpler than the corresponding expression presented by Lancaster and Salkauskas. Then, based on the IMLS method of this paper and the Galerkin weak form, an IEFG method for two-point boundary value problems is presented. Since the shape function of the IMLS method satisfies the property of Kronecker $\delta$ function, then the IEFG method can apply the essential boundary condition directly. And as the number of the coefficients in the trial function of the IMLS method is less than that in the MLS approximation, then fewer nodes are selected in the entire domain in the IEFG method than in the conventional EFG method. Hence, the IEFG method has high computing speed and precision.

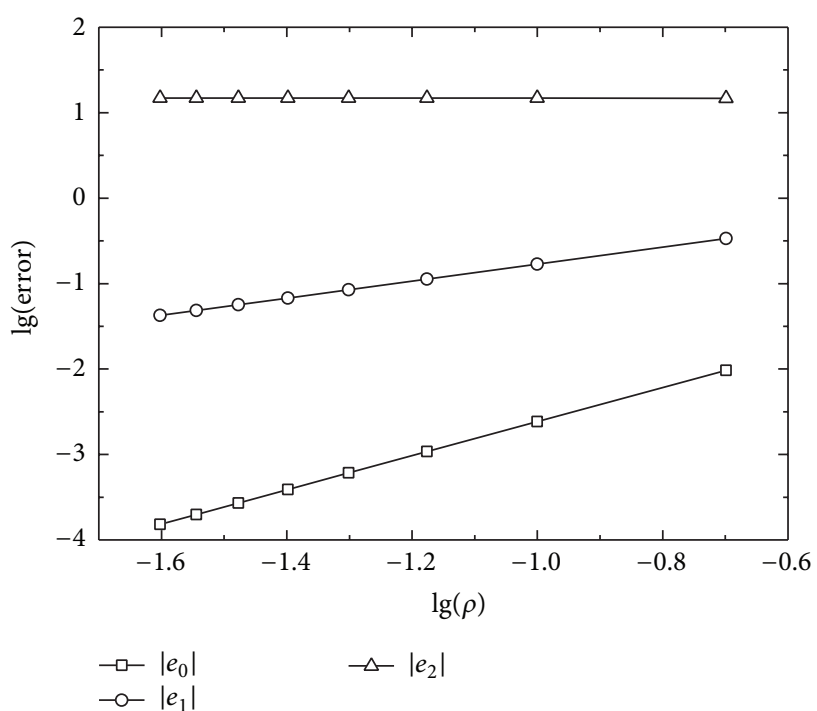

FIGURE 10: Error norms of $\left|e_{0}\right|,\left|e_{1}\right|$, and $\left|e_{2}\right|$ with linear basis functions.

The error analysis of the IEFG method for two-point boundary value problems is presented. The convergence rates of the numerical solution and its derivatives of the IEFG method are presented. The theories of this paper show that if the analytical solution is sufficiently smooth and the order of the polynomial basis functions is big enough, then the solution of the IEFG method and its derivatives are convergent to the analytical solutions in terms of the maximum radius of the domains of influence of nodes. For the purpose of demonstration, some selected numerical examples are given to confirm the theories.

\section{Conflict of Interests}

The authors declare that there is no conflict of interests regarding the publication of this paper.

\section{Acknowledgments}

This work was supported by the National Natural Science Foundation of China (no. 11171208), Shanghai Leading Academic Discipline Project (no. S30106), and the Natural Science Foundation of Ningbo (no. 2013A610103).

\section{References}

[1] K. M. Liew, J. Ren, and J. N. Reddy, "Numerical simulation of thermomechanical behaviours of shape memory alloys via a non-linear mesh-free Galerkin formulation," International Journal for Numerical Methods in Engineering, vol. 63, no. 7, pp. 1014-1040, 2005.

[2] G. R. Liu and Y. T. Gu, "A point interpolation method for two-dimensional solids," International Journal for Numerical Methods in Engineering, vol. 50, no. 4, pp. 937-951, 2001. 
[3] T. Belytschko, Y. Y. Lu, and L. Gu, "Element-free Galerkin methods," International Journal for Numerical Methods in Engineering, vol. 37, no. 2, pp. 229-256, 1994.

[4] W. Ju-Feng, S. Feng-Xin, and C. Rong-Jun, "Element-free Galerkin method for a kind of KdV equation," Chinese Physics $B$, vol. 19, no. 6, Article ID 060201, 2010.

[5] R. J. Cheng and Y. M. Cheng, "A meshless method for the compound KdV-Burgers equation," Chinese Physics B, vol. 20, no. 7, Article ID 070206, 2011.

[6] Y. M. Cheng, M. J. Peng, and J. H. Li, “The complex variable moving least-square approximation and its application," Chinese Journal of Theoretical and Applied Mechanics, vol. 37, no. 6, pp. 719-723, 2005.

[7] Y. M. Cheng and J. H. Li, "A meshless method with complex variables for elasticity," Acta Physica Sinica, vol. 54, no. 10, pp. 4463-4471, 2005.

[8] Y. M. Cheng and J. Li, "A complex variable meshless method for fracture problems," Science in China G: Physics Astronomy, vol. 49, no. 1, pp. 46-59, 2006.

[9] K. M. Liew, C. Feng, Y. M. Cheng, and S. Kitipornchai, "Complex variable moving least-squares method: a meshless approximation technique," International Journal for Numerical Methods in Engineering, vol. 70, no. 1, pp. 46-70, 2007.

[10] J. F. Wang and Y. M. Cheng, "A new complex variable meshless method for transient heat conduction problems," Chinese Physics B, vol. 21, no. 12, Article ID 120206, 2012.

[11] J. F. Wang and Y. M. Cheng, "New complex variable meshless method for advection-diffusion problems," Chinese Physics B, vol. 22, no. 3, Article ID 030208, 2013.

[12] R. J. Cheng and K. M. Liew, "Analyzing two-dimensional sine-Gordon equation with the mesh-free reproducing kernel particle Ritz method," Computer Methods in Applied Mechanics and Engineering, vol. 245-246, pp. 132-143, 2012.

[13] B. D. Dai and Y. M. Cheng, "Local boundary integral equation method based on radial basis functions for potential problems," Acta Physica Sinica, vol. 56, no. 2, pp. 597-603, 2007.

[14] R. J. Cheng and Y. M. Cheng, "A meshless method for solving the inverse heat conduction problem with a source parameter," Acta Physica Sinica, vol. 56, no. 10, pp. 5569-5574, 2007.

[15] R. J. Cheng and Y. M. Cheng, "The meshless method for a two-dimensional inverse heat conduction problem with a source parameter," Chinese Journal of Theoretical and Applied Mechanics, vol. 39, no. 6, pp. 843-847, 2007.

[16] J. F. Wang, F.-N. Bai, and Y. M. Cheng, "A meshless method for the nonlinear generalized regularized long wave equation," Chinese Physics B, vol. 20, no. 3, Article ID 030206, 2011.

[17] S. N. Atluri and T. Zhu, "A new Meshless Local Petrov-Galerkin (MLPG) approach in computational mechanics," Computational Mechanics, vol. 22, no. 2, pp. 117-127, 1998.

[18] L. Chen and Y. M. Cheng, "Reproducing kernel particle method with complex variables for elasticity," Acta Physica Sinica, vol. 57, no. 1, pp. 1-10, 2008.

[19] L. Chen and Y. M. Cheng, "Complex variable reproducing kernel particle method for transient heat conduction problems," Acta Physica Sinica, vol. 57, no. 10, pp. 6047-6055, 2008.

[20] L. Chen and Y. M. Cheng, "The complex variable reproducing kernel particle method for elasto-plasticity problems," Science China: Physics, Mechanics \& Astronomy, vol. 53, no. 5, pp. 954965, 2010.

[21] L. Chen and Y. M. Cheng, "The complex variable reproducing kernel particle method for two-dimensional elastodynamics," Chinese Physics B, vol. 19, no. 9, Article ID 090204, 2010.
[22] Y. J. Weng and Y. M. Cheng, "Analyzing variable coefficient advection-diffusion problems via complex variable reproducing kernel particle method," Chinese Physics B, vol. 22, no. 9, Article ID 090204, 2013.

[23] S. C. Li and Y. M. Cheng, "Meshless numerical manifold method based on unit partition," Acta Mechanica Sinica, vol. 36, no. 4, pp. 496-500, 2004.

[24] S. C. Li and Y. M. Cheng, "Numerical manifold method and its applications in rock mechanics," Advances in Mechanics, vol. 34, no. 4, pp. 446-454, 2004.

[25] S. Li, Y. M. Cheng, and Y.-F. Wu, "Numerical manifold method based on the method of weighted residuals," Computational Mechanics, vol. 35, no. 6, pp. 470-480, 2005.

[26] S. C. Li, S. C. Li, and Y. M. Cheng, "Enriched meshless manifold method for two-dimensional crack modeling," Theoretical and Applied Fracture Mechanics, vol. 44, no. 3, pp. 234-248, 2005.

[27] S.-C. Li, Y. M. Cheng, and S.-C. Li, "Meshless manifold method for dynamic fracture mechanics," Acta Physica Sinica, vol. 55, no. 9, pp. 4760-4766, 2006.

[28] H. F. Gao and Y. M. Cheng, "Complex variable numerical manifold method for elasticity," Chinese Journal of Theoretical and Applied Mechanics, vol. 41, no. 4, pp. 480-488, 2009.

[29] H. Gao and Y. M. Cheng, "A complex variable meshless manifold method for fracture problems," International Journal of Computational Methods, vol. 7, no. 1, pp. 55-81, 2010.

[30] Y. M. Cheng and M. J. Chen, "A boundary element-free method for linear elasticity," Acta Mechanica Sinica, vol. 35, no. 2, pp. 181-186, 2003.

[31] Y. M. Cheng and M. Peng, "Boundary element-free method for elastodynamics," Science in China G: Physics Astronomy, vol. 48, no. 6, pp. 641-657, 2005.

[32] K. M. Liew, Y. M. Cheng, and S. Kitipornchai, "Boundary Element-Free Method (BEFM) for two-dimensional elastodynamic analysis using Laplace transform," International Journal for Numerical Methods in Engineering, vol. 64, no. 12, pp. 16101627, 2005.

[33] S. Kitipornchai, K. M. Liew, and Y. M. Cheng, "A Boundary Element-Free Method (BEFM) for three-dimensional elasticity problems," Computational Mechanics, vol. 36, no. 1, pp. 13-20, 2005.

[34] K. M. Liew, Y. M. Cheng, and S. Kitipornchai, "Boundary Element-Free Method (BEFM) and its application to twodimensional elasticity problems," International Journal for Numerical Methods in Engineering, vol. 65, no. 8, pp. 1310-1332, 2006.

[35] Y.-X. Qin and Y. M. Cheng, "Reproducing kernel particle boundary element-free method," Acta Physica Sinica, vol. 55, no. 7, pp. 3215-3222, 2006.

[36] K. M. Liew, Y. M. Cheng, and S. Kitipornchai, "Analyzing the $2 \mathrm{D}$ fracture problems via the enriched boundary element-free method," International Journal of Solids and Structures, vol. 44, no. 11-12, pp. 4220-4233, 2007.

[37] Y. X. Qin and Y. M. Cheng, "Reproducing kernel particle boundary element-free method for potential problems," Chinese Journal of Theoretical and Applied Mechanics, vol. 41, no. 6, pp. 898-905, 2009.

[38] K. M. Liew and Y. M. Cheng, "Complex variable boundary element-free method for two-dimensional elastodynamic problems," Computer Methods in Applied Mechanics and Engineering, vol. 198, no. 49-52, pp. 3925-3933, 2009. 
[39] M. Peng and Y. M. Cheng, "A Boundary Element-Free Method (BEFM) for two-dimensional potential problems," Engineering Analysis with Boundary Elements, vol. 33, no. 1, pp. 77-82, 2009.

[40] Y. M. Cheng, K. M. Liew, and S. Kitipornchair, "Reply to "Comments on Boundary Element-Free Method (BEFM) and its application to two-dimensional elasticity problems"', International Journal for Numerical Methods in Engineering, vol. 78, no. 10, pp. 1258-1260, 2009.

[41] S. N. Atluri, J. Sladek, V. Sladek, and T. Zhu, "The Local Boundary Integral Equation (LBIE) and it's meshless implementation for linear elasticity," Computational Mechanics, vol. 25, no. 2-3, pp. 180-198, 2000.

[42] B. Dai and Y. M. Cheng, "An improved local boundary integral equation method for two-dimensional potential problems," International Journal of Applied Mechanics, vol. 2, no. 2, pp. 421436, 2010.

[43] Z. Zhang, K. M. Liew, Y. M. Cheng, and Y. Y. Lee, "Analyzing 2D fracture problems with the improved element-free Galerkin method," Engineering Analysis with Boundary Elements, vol. 32, no. 3, pp. 241-250, 2008.

[44] Z. Zhang, K. M. Liew, and Y. M. Cheng, "Coupling of the improved element-free Galerkin and boundary element methods for two-dimensional elasticity problems," Engineering Analysis with Boundary Elements, vol. 32, no. 2, pp. 100-107, 2008.

[45] Z. Zhang, D.-M. Li, Y. M. Cheng, and K. M. Liew, "The improved element-free Galerkin method for three-dimensional wave equation," Acta Mechanica Sinica, vol. 28, no. 3, pp. 808818, 2012.

[46] Z. Zhang, J. F. Wang, Y. M. Cheng, and K. M. Liew, “The improved element-free Galerkin method for three-dimensional transient heat conduction problems," Science China: Physics, Mechanics \& Astronomy, vol. 56, no. 8, pp. 1568-1580, 2013.

[47] Z. Zhang, S. Y. Hao, K. M. Liew, and Y. M. Cheng, "The improved element-free Galerkin method for two-dimensional elastodynamics problems," Engineering Analysis with Boundary Elements, vol. 37, no. 12, pp. 1576-1584, 2013.

[48] M. J. Peng, R. X. Li, and Y. M. Cheng, "Analyzing threedimensional viscoelasticity problems via the Improved Element-Free Galerkin (IEFG) method," Engineering Analysis with Boundary Elements, vol. 40, pp. 104-113, 2014.

[49] M. Peng, P. Liu, and Y. M. Cheng, "The Complex Variable Element-Free Galerkin (CVEFG) method for two-dimensional elasticity problems," International Journal of Applied Mechanics, vol. 1, no. 2, pp. 367-385, 2009.

[50] M. Peng, D. Li, and Y. M. Cheng, "The Complex Variable Element-Free Galerkin (CVEFG) method for elasto-plasticity problems," Engineering Structures, vol. 33, no. 1, pp. 127-135, 2011.

[51] Y. M. Cheng, J. F. Wang, and F. N. Bai, "A new complex variable element-free Galerkin method for two-dimensional potential problems," Chinese Physics B, vol. 21, no. 9, Article ID 090203, 2012.

[52] Y. M. Cheng, R. X. Li, and M. J. Peng, "Complex Variable Element-Free Galerkin (CVEFG) method for viscoelasticity problems," Chinese Physics B, vol. 21, no. 9, Article ID 090205, 2012.

[53] Y. M. Cheng, J. F. Wang, and R. X. Li, “The Complex Variable Element-Free Galerkin (CVEFG) method for two-dimensional elastodynamics problems," International Journal of Applied Mechanics, vol. 4, no. 4, Article ID 125004, 23 pages, 2012.
[54] D. Li, F. Bai, Y. M. Cheng, and K. M. Liew, "A novel complex variable element-free Galerkin method for two-dimensional large deformation problems," Computer Methods in Applied Mechanics and Engineering, vol. 233-236, pp. 1-10, 2012.

[55] F.-N. Bai, D.-M. Li, J. F. Wang, and Y. M. Cheng, "An improved complex variable element-free Galerkin method for two-dimensional elasticity problems," Chinese Physics B, vol. 21, no. 2, Article ID 020204, 2012.

[56] D. M. Li, K. M. Liew, and Y. M. Cheng, "An improved complex variable element-free Galerkin method for two-dimensional large deformation elastoplasticity problems," Computer Methods in Applied Mechanics and Engineering, vol. 269, pp. 72-86, 2014.

[57] T. Zhu and S. N. Atluri, "A modified collocation method and a penalty formulation for enforcing the essential boundary conditions in the element free Galerkin method," Computational Mechanics, vol. 21, no. 3, pp. 211-222, 1998.

[58] T. Most and C. Bucher, "A moving least squares weighting function for the element-free Galerkin method which almost fulfills essential boundary conditions," Structural Engineering and Mechanics, vol. 21, no. 3, pp. 315-332, 2005.

[59] T. Most and C. Bucher, "New concepts for moving least squares: an interpolating non-singular weighting function and weighted nodal least squares," Engineering Analysis with Boundary Elements, vol. 32, no. 6, pp. 461-470, 2008.

[60] P. Lancaster and K. Salkauskas, "Surfaces generated by moving least squares methods," Mathematics of Computation, vol. 37, no. 155 , pp. 141-158, 1981.

[61] I. Kaljević and S. Saigal, "An improved element free Galerkin formulation," International Journal for Numerical Methods in Engineering, vol. 40, no. 16, pp. 2953-2974, 1997.

[62] H.-P. Ren, Y. M. Cheng, and W. Zhang, "An Improved Boundary Element-Free Method (IBEFM) for two-dimensional potential problems," Chinese Physics B, vol. 18, no. 10, pp. 4065-4073, 2009.

[63] R. Hongping, C. Yumin, and Z. Wu, "An Interpolating Boundary Element-Free Method (IBEFM) for elasticity problems," Science China: Physics, Mechanics \& Astronomy, vol. 53, no. 4, pp. 758-766, 2010.

[64] H. P. Ren and Y. M. Cheng, "The Interpolating ElementFree Galerkin (IEFG) method for two-dimensional elasticity problems," International Journal of Applied Mechanics, vol. 3, no. 4, pp. 735-758, 2011.

[65] H. Ren and Y. M. Cheng, "The Interpolating Element-Free Galerkin (IEFG) method for two-dimensional potential problems," Engineering Analysis with Boundary Elements, vol. 36, no. 5, pp. 873-880, 2012.

[66] J. F. Wang, F. X. Sun, and Y. M. Cheng, "An improved interpolating element-free Galerkin method with nonsingular weight function for two-dimensional potential problems," Chinese Physics B, vol. 21, no. 9, Article ID 090204, 2012.

[67] J. Wang, J. Wang, F. Sun, and Y. M. Cheng, "An interpolating boundary element-free method with nonsingular weight function for two-dimensional potential problems," International Journal of Computational Methods, vol. 10, no. 6, Article ID 1350043, 23 pages, 2013.

[68] F. X. Sun, J. F. Wang, and Y. M. Cheng, "An improved interpolating element-free Galerkin method for elasticity," Chinese Physics B, vol. 22, no. 12, Article ID 120203, 2013.

[69] D. Levin, "The approximation power of moving least-squares," Mathematics of Computation, vol. 67, no. 224, pp. 1517-1531, 1998. 
[70] M. G. Armentano and R. G. Durán, “Error estimates for moving least square approximations," Applied Numerical Mathematics, vol. 37, no. 3, pp. 397-416, 2001.

[71] C. Zuppa, "Error estimates for moving least square approximations," Bulletin of the Brazilian Mathematical Society, vol. 34, no. 2, pp. 231-249, 2003.

[72] X. Li and J. Zhu, "A Galerkin boundary node method and its convergence analysis," Journal of Computational and Applied Mathematics, vol. 230, no. 1, pp. 314-328, 2009.

[73] X. Li, "Meshless Galerkin algorithms for boundary integral equations with moving least square approximations," Applied Numerical Mathematics, vol. 61, no. 12, pp. 1237-1256, 2011.

[74] X. Li, "The meshless Galerkin boundary node method for Stokes problems in three dimensions," International Journal for Numerical Methods in Engineering, vol. 88, no. 5, pp. 442-472, 2011.

[75] P. Krysl and T. Belytschko, "Element-free Galerkin method: convergence of the continuous and discontinuous shape functions," Computer Methods in Applied Mechanics and Engineering, vol. 148, no. 3-4, pp. 257-277, 1997.

[76] H.-J. Chung and T. Belytschko, "An error estimate in the EFG method," Computational Mechanics, vol. 21, no. 2, pp. 91-100, 1998.

[77] J. Dolbow and T. Belytschko, "Numerical integration of the Galerkin weak form in meshfree methods," Computational Mechanics, vol. 23, no. 3, pp. 219-230, 1999.

[78] L. Gavete, J. L. Cuesta, and A. Ruiz, "A procedure for approximation of the error in the EFG method," International Journal for Numerical Methods in Engineering, vol. 53, no. 3, pp. 677-690, 2002.

[79] L. Gavete, M. L. Gavete, B. Alonso, and A. J. Martín, "A posteriori error approximation in EFG method," International Journal for Numerical Methods in Engineering, vol. 58, no. 15, pp. 2239-2263, 2003.

[80] R. J. Cheng and Y. M. Cheng, "Error estimates for the finite point method," Applied Numerical Mathematics, vol. 58, no. 6, pp. 884-898, 2008.

[81] R. J. Cheng and Y. M. Cheng, "Error estimates of the elementfree Galerkin method for potential problems," Acta Physica Sinica, vol. 57, no. 10, pp. 6037-6046, 2008.

[82] R. J. Cheng and Y. M. Cheng, "Error estimate of element-free Galerkin method for elasticity," Acta Physica Sinica, vol. 60, no. 7, Article ID 070206, 2011. 


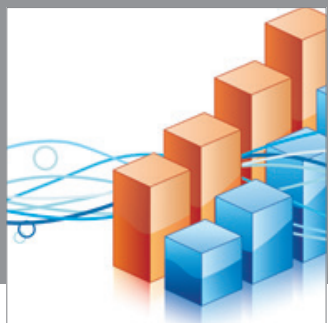

Advances in

Operations Research

mansans

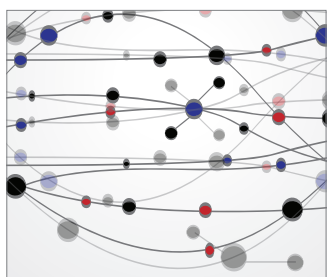

The Scientific World Journal
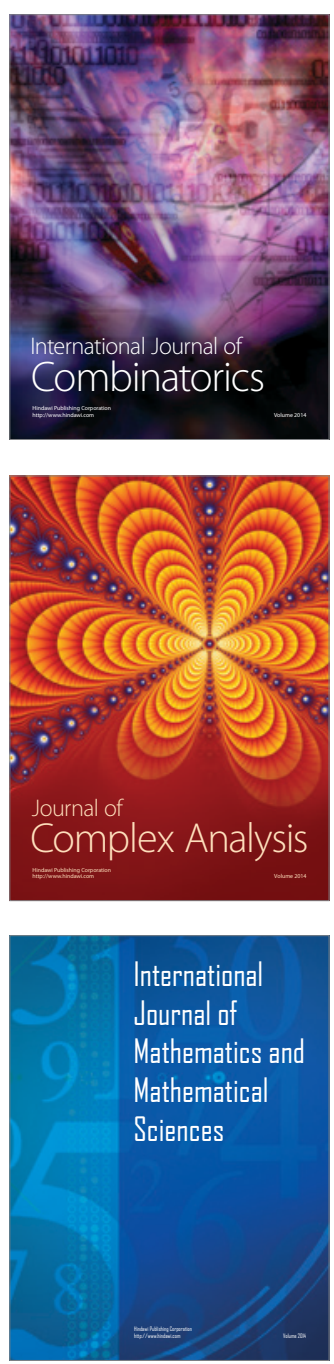
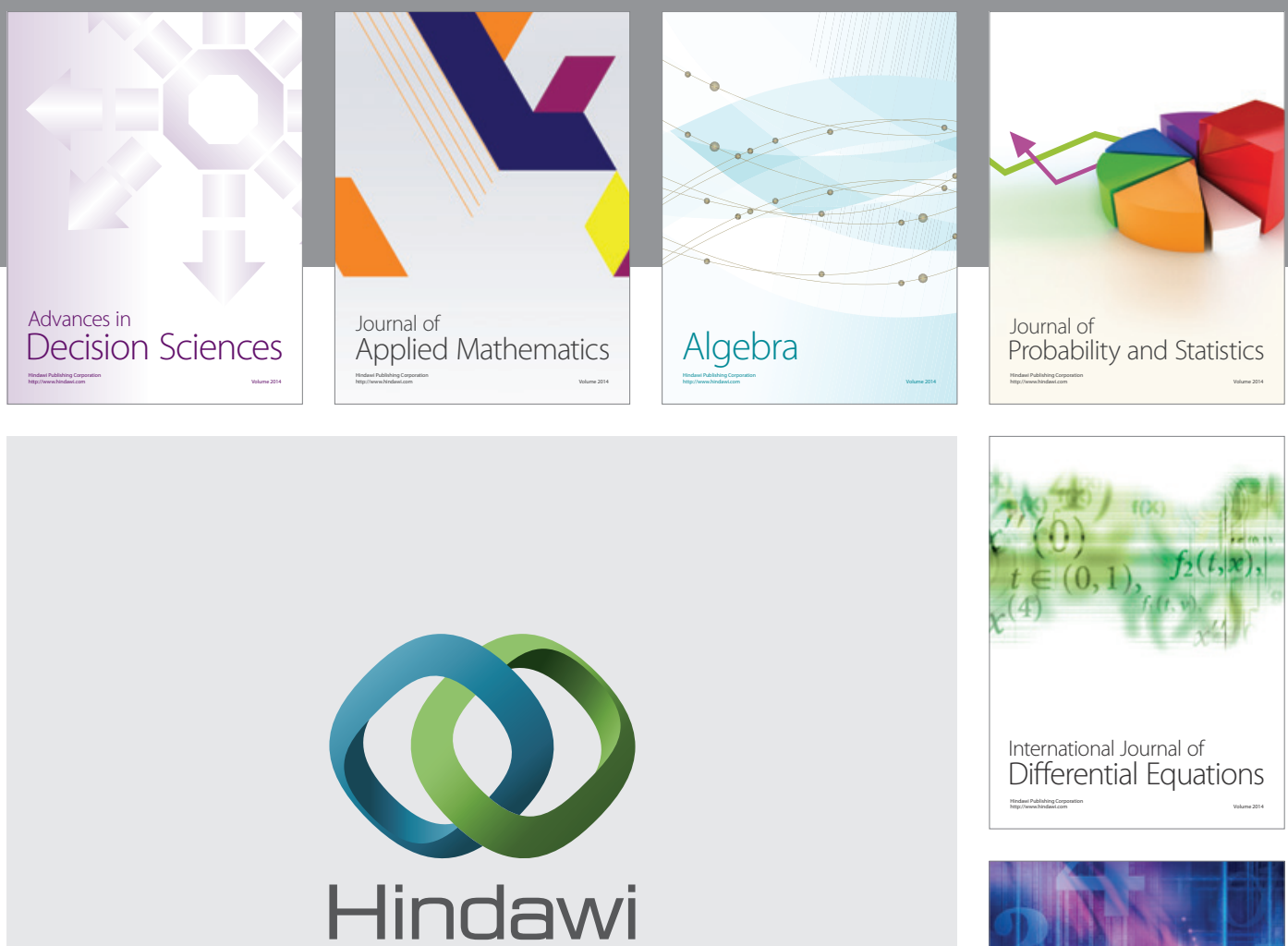

Submit your manuscripts at http://www.hindawi.com
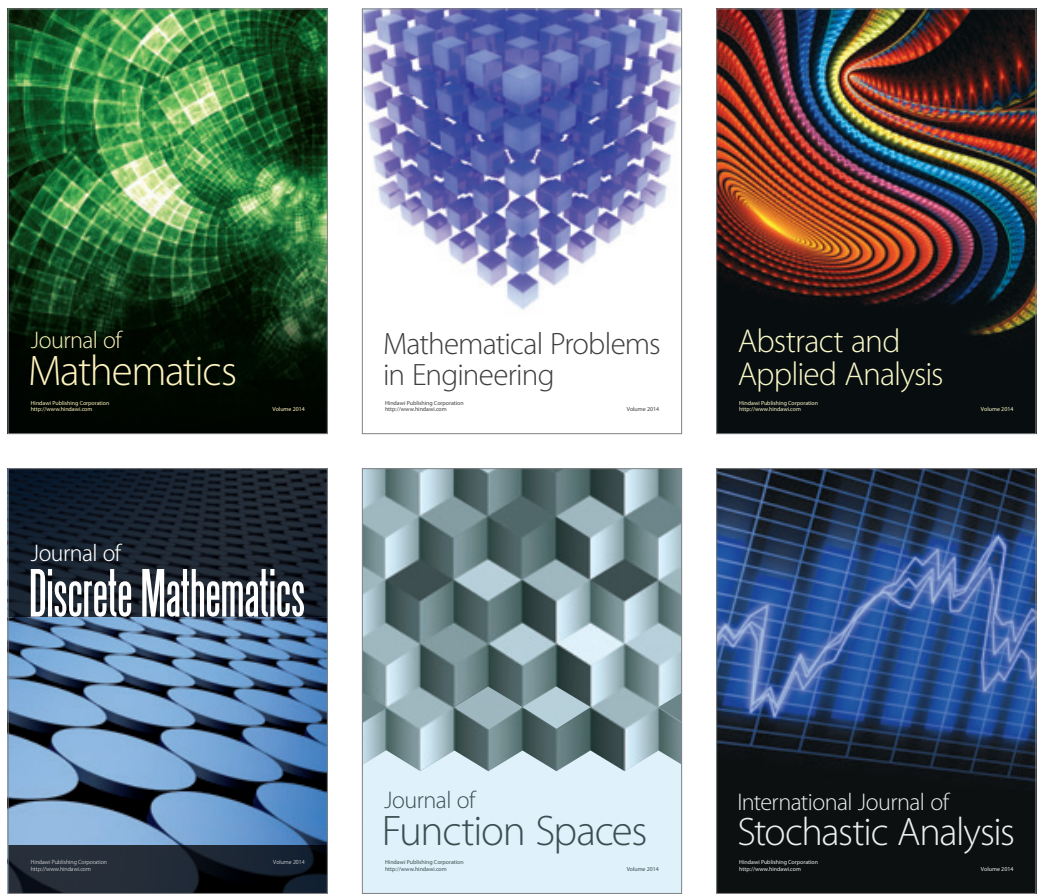

Journal of

Function Spaces

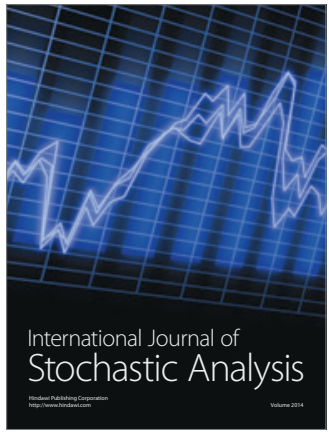

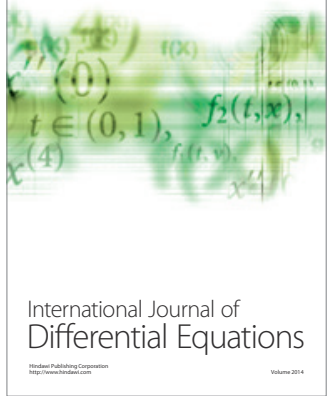
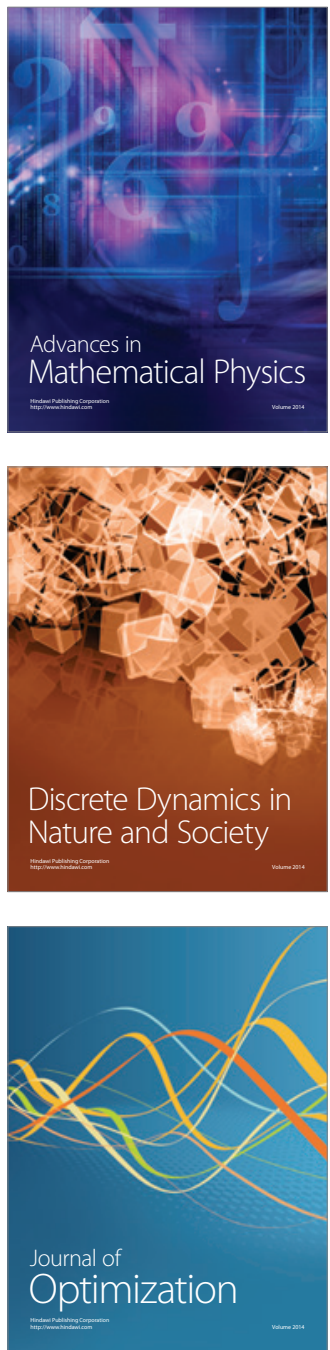